\title{
Ultra-High Q Silicon Gyroscopes with Interchangeable Rate and Whole Angle Modes of Operation
}

\author{
Alexander A. Trusov, Igor P. Prikhodko, Sergei A. Zotov, Adam R. Schofield, Andrei M. Shkel \\ MicroSystems Laboratory, University of California, Irvine, CA, USA \\ Email: \{atrusov, iprikhod, szotov, aschofie, ashkel\}@uci.edu
}

\begin{abstract}
We report a new family of ultra-high Q silicon MEMS tuning fork gyroscopes demonstrating angle rate and, for the first time, rate integrating (whole angle) operation. The novel mechanical architecture eliminates low frequency in-phase modes and maximizes the Q-factors. A vacuum packaged SOI dual mass gyroscope with a $1.7 \mathrm{kHz}$ operational frequency demonstrated drive- and sense- mode Q-factors of 0.31 and 0.64 million, respectively. A completely symmetric, dynamically balanced quadruple mass gyroscope with a $2.2 \mathrm{kHz}$ operational frequency demonstrated identical drive- and sense-mode Q-factors of 0.45 million. Due to the stiffness and damping symmetry, the new gyroscope can be instrumented to measure the angle of rotation directly, eliminating the bandwidth and dynamic range limitations of conventional MEMS vibratory rate gyroscopes. The technology may enable silicon micromachined devices for inertial guidance applications previously limited to precision-machined quartz hemispherical resonator gyroscopes.
\end{abstract}

\section{INTRODUCTION}

The operation of micromachined vibratory gyroscopes is based on a transfer of energy between two modes of vibration caused by the Coriolis effect [1]. Maximization of the driveand sense-mode quality factors (Q-factors) is key to enhancing performance of micromachined vibratory gyroscopes [2]. Mode-matched silicon tuning fork gyroscopes with Q-factors on the order of 100 thousand have been demonstrated to achieve sub-degree-per-hour bias stability in a $0.1 \mathrm{~Hz}$ measurement bandwidth [3]. However, mode matching of a high-Q vibratory gyroscope operated in rate measuring mode fundamentally limits the linear range to below $100 \mathrm{deg} / \mathrm{s}$, and constrains the rate measurement bandwidth to less than $1 \mathrm{~Hz}$. These limitations of conventional high-Q vibratory rate micromachined gyroscopes can be eliminated by operating the gyroscope structure in the rate integrating, or whole angle mode [4].

An ideal angle measuring gyroscope, such as the classical macro-scale Foucault pendulum, is based on a highly isotropic resonator. The successful realization of a micromachined implementation requires a mechanical structure with highly symmetric mode-shapes providing matched frequency and Qfactors over a practical temperature range. Since the operation of an angle measuring gyroscope is based on the free precession of the inertial mass [1], maximization of the energy

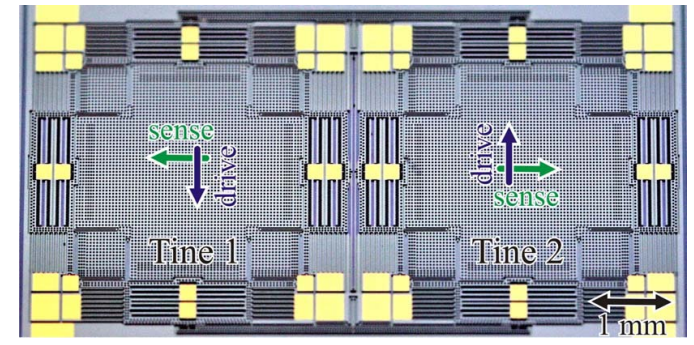

Figure 1. Photograph of a fabricated dual mass anti-phase gyroscope.

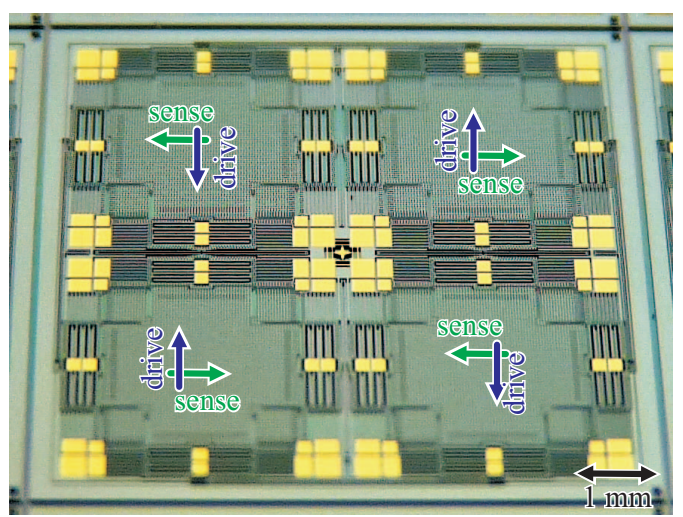

Figure 2. Photograph of a fabricated quadruple mass anti-phase gyroscope.

dissipation time constant is also extremely critical. Previously reported MEMS gyroscopes, however, suffer from mismatches in frequency and damping, and short energy dissipation constants of less than 1 second ( $0.1 \mathrm{~s}$ typical) making them unfavorable for angle measuring operation.

This paper reports a new family of vacuum packaged, dynamically balanced silicon micromachined gyroscopes achieving free vibrations decay time constants on the order of 1 minute enabling interchangeable operation in rate and whole angle modes, Fig. 1 and 2. Dissipation of energy through the substrate is minimized by the means of complete anti-phase operation facilitated by an integrated mechanical lever mechanism [5]. Operation at low frequencies (on the order of $1 \mathrm{kHz}$ ) decouples the mechanical resonance and thermal relaxation time constants, thereby increasing the fundamental thermoelastic Q-factor limit above 1 million.

This work was funded by ONR/NSWCDD grant no N00014-09-1-0424. 


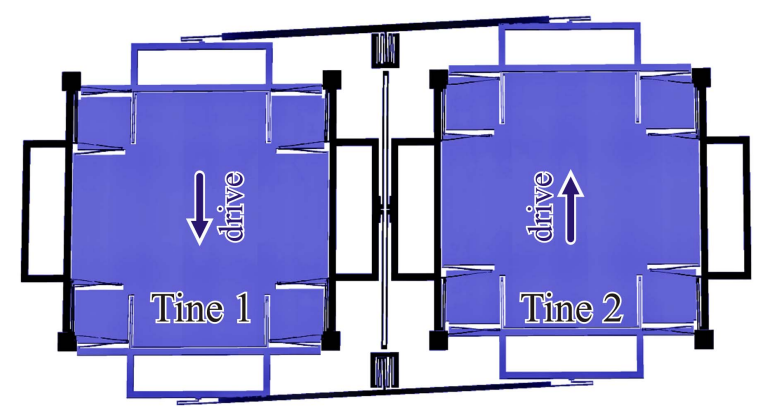

Figure 3. Dual mass gyroscope operation: levered drive-mode with anti-phase synchronization and linear momentum balancing, modeling.

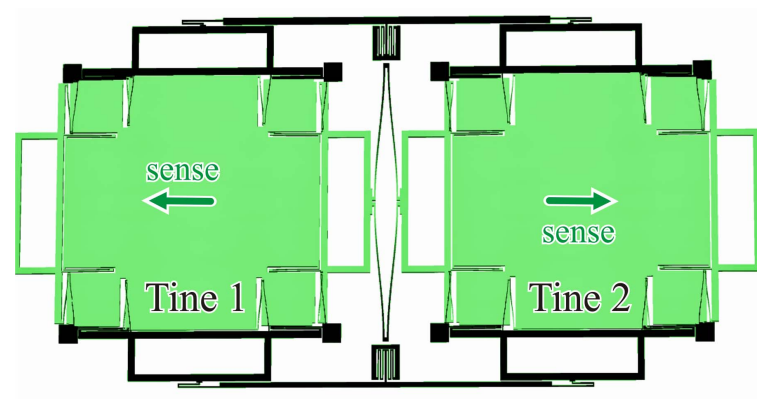

Figure 4. Dual mass gyroscope operation: linearly coupled anti-phase sense-mode with complete dynamic balance for $\mathrm{Q}$ maximization, modeling.

\section{DESIGN CONCEPT}

In this section we describe the mechanical architectures of the anti-phase operated dual and quadruple mass gyroscopes.

\section{A. Dual Mass Gyroscope}

The mechanical architecture of the dual mass gyroscope, Fig. 1, comprises two identical symmetrically decoupled tines [6], a drive-mode anti-phase synchronization lever mechanism [5], and coupling flexures for the linear anti-phase sense-mode. Each tine is an isotropic resonator consisting of a square proof mass suspended by two drive-mode and two sense-mode decoupling shuttles. The drive-mode of the dual mass gyroscope is formed by the two tines forced into antiparallel, anti-phase motion synchronized by the integrated mechanical lever system, Fig. 3. The sense-mode of the dual mass gyroscope is formed by the two linearly coupled tines moving in anti-phase, Fig. 4.

For rate measuring mode of operation, the gyroscope is continuously driven into anti-phase motion using lateral comb electrodes on the drive-mode shuttles. Rotation around the sensitive z-axis results in Coriolis excitation of the linear antiphase sense-mode, which is detected capacitively using differential parallel plate electrodes on the sense-mode decoupling shuttles. Unlike conventional tuning fork gyroscopes, this dual mass architecture prioritizes the quality factor of the sense-mode by mechanical design. While the intentional mode arrangement maximizes the angular rate sensitivity, the inherent non-symmetry of dual mass tuning fork gyroscopes limits the ability to match drive- and sensemode Q-factors in vacuum as required for the rate integrating (whole angle) mode of operation.

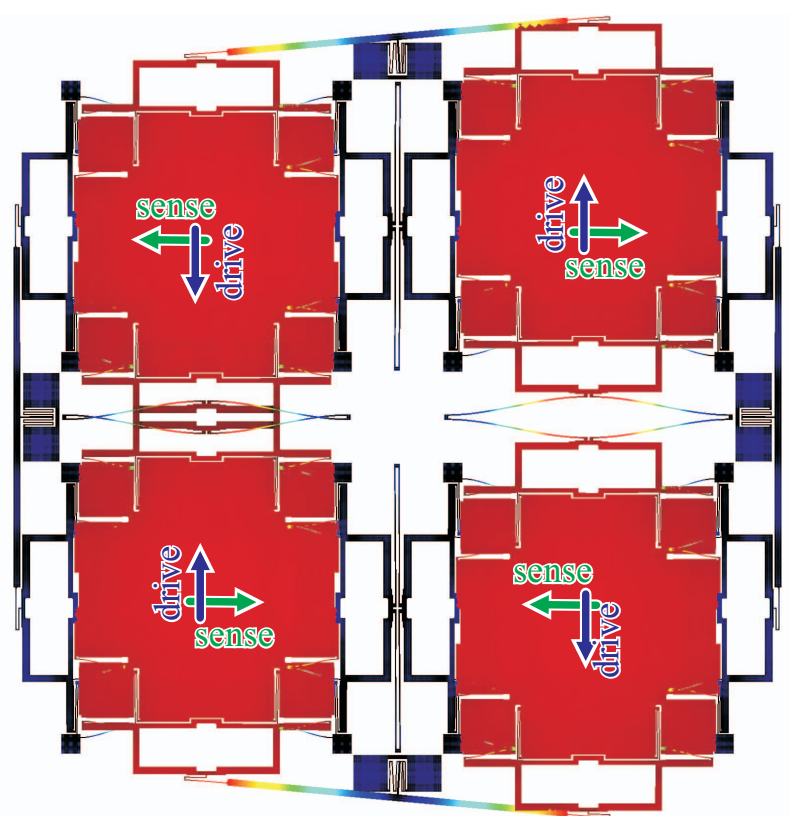

Figure 5. Quadruple mass gyroscope operation: degenerate, anti-phase drive- and sense-modes provide complete dynamic balance, low dissipation of energy, and matching of frequency and Q-factor by mechanical design.

\section{B. Quadruple Mass Gyroscope}

Similarly to the dual mass device, the mechanical structure of the quadruple mass gyroscope, Fig. 2, comprises four identical symmetrically decoupled tines, a pair of anti-phase synchronization lever mechanisms for both the drive- and the sense-mode, and linear coupling flexures between the tines. The architecture of the quadruple mass gyroscope builds upon the dual mass design and can be thought of as two levered dual mass tuning fork gyroscopes coupled together with additional linear flexures and anti-phase lever mechanisms. Operation of the quadruple mass gyroscope is illustrated by the Finite Element Modeling of the drive-mode in Fig. 5. Due to the symmetry of the device, the drive- and sense-modes are degenerate and spatially oriented at a $90^{\circ}$ angle.

The quadruple mass tuning fork preserves and expands the structural advantages of the levered dual mass design. Unlike conventional tuning fork devices, the levered quadruple mass architecture provides true mechanical rejection of external vibrations and shocks along both the drive and sense axes. Structural symmetry of the device suggests improved robustness to fabrication imperfections and temperature induced frequency drifts, which are known sources of drifts in high-Q vibratory gyroscopes. Most importantly, the quadruple mass design is expected to enable whole angle (rate integrating) mode of operation due to its unique combination of low energy dissipation and isotropy of both the resonant frequency and damping.

\section{EXPERIMENTAL CHARACTERIZATION}

In this section we experimentally evaluate the dual and quadruple mass gyroscope design hypotheses using in-house fabricated and vacuum packaged prototypes. 


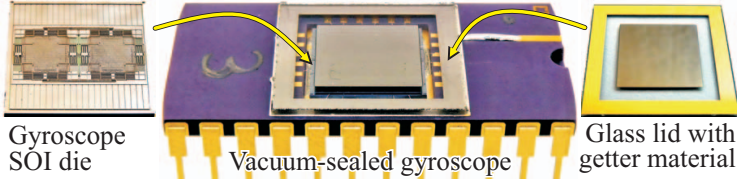

Figure 6. Photograph of a vacuum packaged gyroscope, showing a gyro die, ceramic DIP-24 package, and custom made glass lid with getter material.

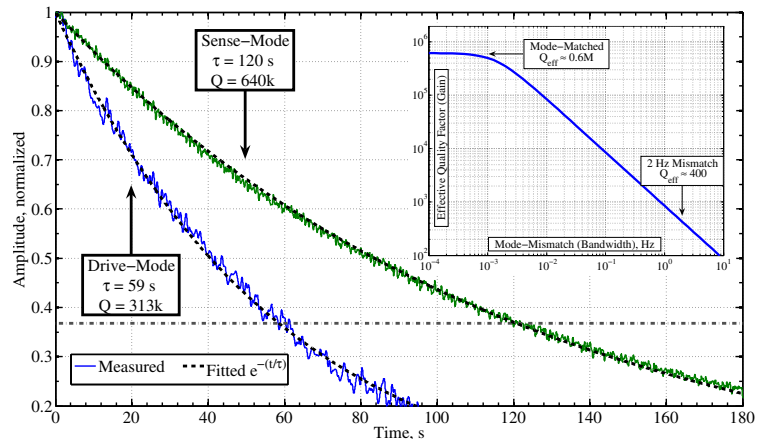

Figure 7. Measurement of packaged gyroscope Q-factors using ring-down tests. Drive- and sense-mode Q-factor is 0.31 and 0.64 million, respectively. Inset: analytical modeling of gain-bandwidth characteristic of the gyroscope.

\section{A. Fabrication and Vacuum Packaging}

The fabrication of prototypes was done using an in-house, wafer-level, single-mask process based on silicon-on-insulator (SOI) substrates with $50 \mu \mathrm{m}$ and $100 \mu \mathrm{m}$ thick device layer and a $5 \mu \mathrm{m}$ buried oxide layer. A hard mask was defined on the structural layer by depositing, patterning, and dry etching a $1 \mu \mathrm{m}$ thick surface oxide layer. Devices were defined in the highly doped structural layer by DRIE. Finally, the perforated structures were released using a timed $20 \% \mathrm{HF}$ acid bath. A package-level technology for the robust vacuum sealing of the high-Q gyroscopes was implemented to allow experimental characterization of standalone sensors, Fig. 6. The packaging procedure comprises the eutectic attachment of a gyroscope die to a ceramic package, wirebonding, and eutectic sealing of the device in a $10^{-6}$ Torr vacuum preceded by activation of the getter deposited on the lid [7].

\section{B. Dual Mass Gyroscope Characterization}

Ring-down characterization of a vacuum packaged dual mass gyroscope with a $1.7 \mathrm{kHz}$ drive-mode operational frequency revealed a measured drive-mode Q-factor of 0.31 million and a sense-mode Q-factor of 0.64 million, Fig. 7. To achieve the best rate sensitivity, a vacuum packaged gyroscope should be operated under a close matching between the drive- and the sense-modes maintained over a practical range. Fig. 8 shows measured frequency-temperature characteristics of the drive- and sense-modes, revealing linear TCFs of $-22 \mathrm{ppm} /{ }^{\circ} \mathrm{C}$ and $-28 \mathrm{ppm} /{ }^{\circ} \mathrm{C}$, respectively. Preliminary open loop rate characterization of the gyroscope was performed with a $7 \mathrm{~Hz}$ frequency mismatch, Fig. 9. From the gain-bandwidth analysis in Fig. 7, the rate sensitivity is expected to improve by $+40 \mathrm{~dB}$ for $0.1 \mathrm{~Hz}$ mode matching, and by $+60 \mathrm{~dB}$ for $0.01 \mathrm{~Hz}$ matching. The dual mass gyroscope experimental results confirm the ultra-high $\mathrm{Q}$ design hypothesis and motivate the investigation of the completely balanced, symmetric quadruple mass gyroscope.

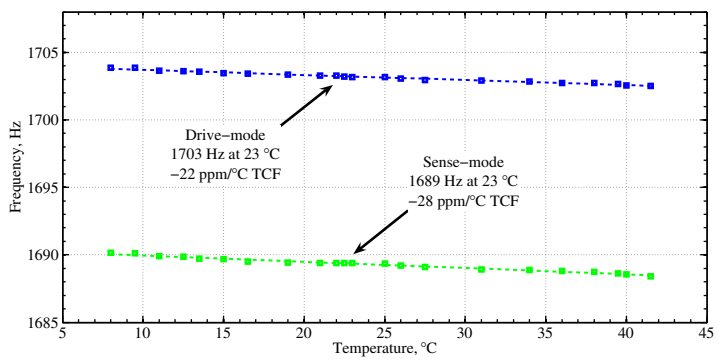

Figure 8. Measured frequency versus temperature for the vacuum packaged dual mass gyroscope revealed a linear TCF of approximately $-25 \mathrm{ppm} /{ }^{\circ} \mathrm{C}$.

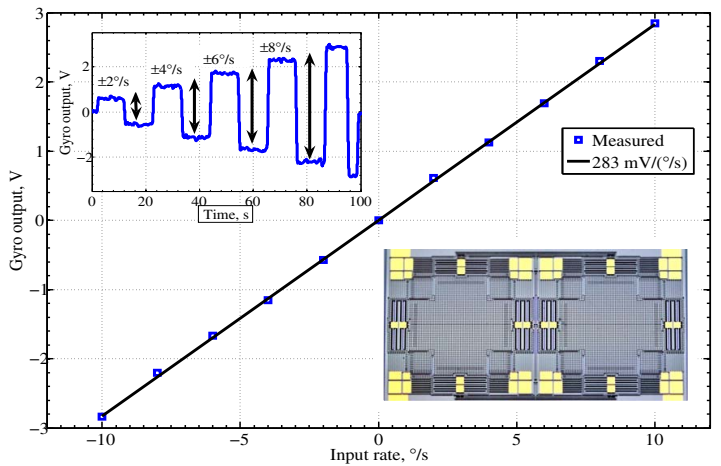

Figure 9. Experimental rate characterization of the dual mass gyroscope under a $7 \mathrm{~Hz}$ mismatch between the drive- and sense-mode resonances.

\section{Quadruple Mass Gyroscope Characterization}

Structural characterization of a $2,180 \mathrm{~Hz}$ quadruple mass gyroscope operated in vacuum is shown in Fig. 10. The ringdown measurements revealed an identical drive- and sensemode Q-factor of 0.45 million, yielding an isotropic energy dissipation time constant in excess of 1 minute. The effect of temperature on the drive- and sense-mode frequency and Qfactor was characterized using a thermal chamber, Fig. 11. The experiments revealed a linear dependency of the frequencies on temperature with an identical TCF of $-22.5 \pm 0.27 \mathrm{ppm} /{ }^{\circ} \mathrm{C}$. Isotropic Q-factors above 0.38 million were experimentally observed for temperatures up to $75^{\circ} \mathrm{C}$.

The quadruple mass gyroscope was characterized in the angle rate and rate integrating (whole angle) modes of operation. For the angle rate operation, the structure was continuously driven into drive-mode anti-phase vibrations, while the Coriolis induced response of the sense-mode was detected to produce a rate output. In this mode of operation, the quadruple mass gyroscope's characteristics are similar to the dual mass device. To experimentally evaluate the rate integrating (whole angle) feasibility, the vacuum operated quadruple mass gyroscope was first mode-matched to within $20 \mathrm{mHz}$ and initiated into anti-phase drive-mode resonance using an off-chip PLL. Upon achieving amplitude of several micrometers, all the driving voltages were switched off to enable free precession of the ultra-high $\mathrm{Q}$ isotropic resonator. Orientation of the free vibrations pattern remains fixed in inertial space, providing a direct measurement of the rotation angle in a wide bandwidth and range, Fig. 12. Characterization of the mode-matched quadruple mass device operated in free vibrations regime demonstrated rate integrating (whole angle) mode of operation, confirming the design hypotheses. 


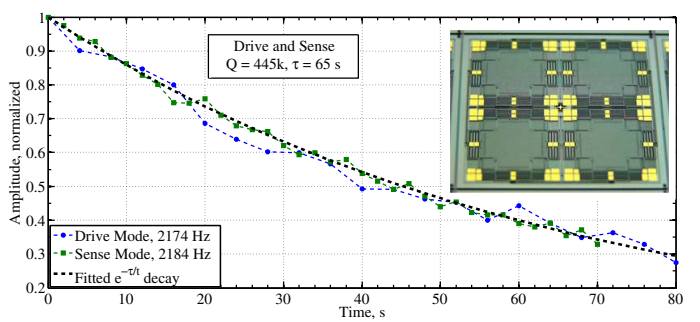

Figure 10. Measurement of the quadruple mass gyroscope Q-factors using ring-down tests. Both drive- and sense-mode Q-factor is 0.45 million.

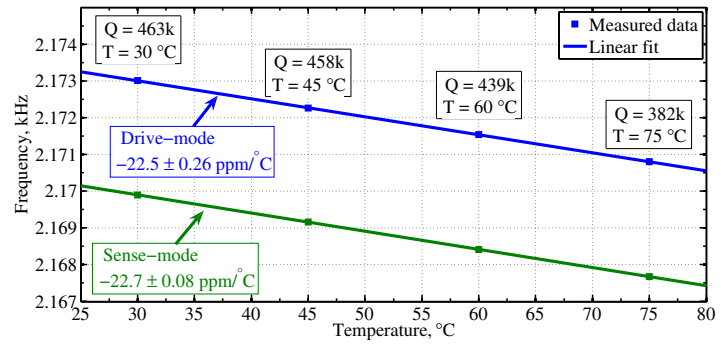

Figure 11. Measured frequency and $\mathrm{Q}$ versus temperature for the quadruple mass gyroscope. Drive and sense have a matched TCF of $-22.5 \mathrm{ppm} /{ }^{\circ} \mathrm{C}$.

\section{CONCLUSIONS}

We report a new family of silicon MEMS tuning fork gyroscopes enabling, for the first time, interchangeable operation in rate and rate integrating (whole angle) regimes. A vacuum packaged dual mass SOI gyroscope with a $1.7 \mathrm{kHz}$ operational frequency demonstrated drive- and sense- mode Q-factors of 0.31 and 0.64 million, respectively. A completely symmetric, dynamically balanced quadruple mass gyroscope with a $2.2 \mathrm{kHz}$ operational frequency demonstrated identical drive- and sense-mode Q-factors of 0.45 million. The low energy dissipation allows reducing the driving voltages to $1 \mathrm{mV}$ AC (with $1 \mathrm{~V}$ DC polarization), and provides mechanical sensitivity on the order of $1 \mathrm{~nm} /(\% / \mathrm{h})$ with a fundamental mechanical-thermal resolution limit on the order of $0.01 \% / \mathrm{h} / \sqrt{ } \mathrm{Hz}$. The measured frequency-temperature characteristics of the gyroscopes are linear with identical TCFs, making them more suitable for electrostatic compensation than quadratic dependency of high-Q quartz devices [8]. Unlike most high-Q silicon devices, the gyroscopes can also be ovenized [9] without a significant performance sacrifice since Q-factors on the order of 0.3 million are sustained up to $75^{\circ} \mathrm{C}$. Due to the stiffness and damping symmetry, the quadruple mass gyroscope can be instrumented to measure the angle of rotation directly, eliminating the bandwidth and dynamic range limitations of conventional MEMS vibratory rate gyroscopes. The technology may enable silicon micromachined devices for inertial guidance applications previously limited to precisionmachined quartz hemispherical resonator gyroscopes.

\section{ACKNOWLEDGMENT}

The authors would like to acknowledge Tom Lee and Joe Zendejas of the UCLA Nanoelectronics Research Facility for assistance with the fabrication of prototypes, Heather Florence of SAES Getters and Paul Barnes of SST International for

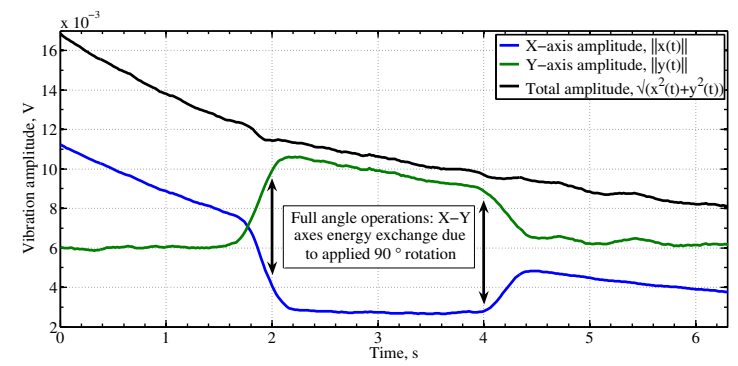

(a) Exchange of energy between the two modes in response to $90^{\circ}$ rotations.

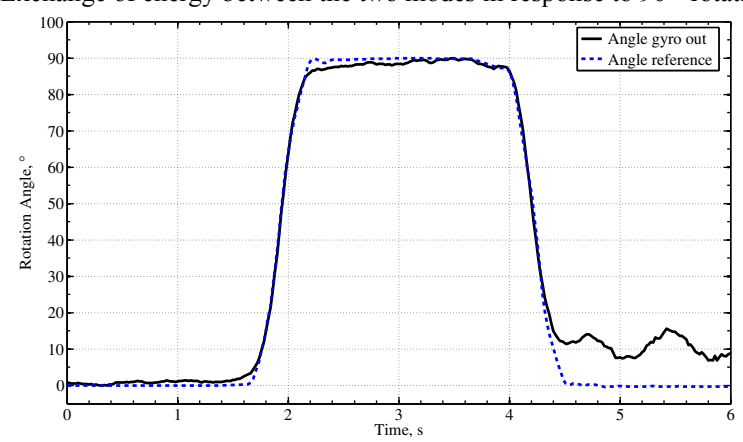

(b) Angle of rotation measured directly from the proof mass precession.

Figure 12. Experimental demonstration of the rate integrating (whole angle) operation using free vibrations of the ultra-high Q four mass gyroscope.

assistance with vacuum packaging, and Ilya Chepurko for assistance with the interface electronics. The gyroscopes were designed and characterized at the MicroSystems Laboratory, University of California, Irvine.

\section{REFERENCES}

[1] A.M. Shkel, "Type I and type II micromachined vibratory gyroscopes," in Proc. IEEE/ION PLANS, San Diego, CA, USA, April 24-27, 2006.

[2] M. Weinberg, R. Candler, S. Chandorkar, J. Varsanik, T. Kenny, A. Duwel, "Energy loss in MEMS resonators and the impact on inertial and RF devices," TRANSDUCERS 2009, Denver, CO, USA, 2009.

[3] M.F. Zaman, A. Sharma, Z. Hao, F. Ayazi, "A mode-matched siliconyaw tuning-fork gyroscope with subdegree-per-hour Allan deviation bias instability," JMEMS, vol.17, no.6, pp.1526-1536, December 2008.

[4] A.M. Shkel, C. Acar, C. Painter, "Two types of micromachined vibratory gyroscopes," pp. 531-536, 2005 IEEE Sensors, Irvine, CA, USA.

[5] A.A. Trusov, A.R. Schofield, A.M. Shkel, "Gyroscope architecture with structurally forced anti-phase drive-mode and linearly coupled anti-phase sense-mode," TRANSDUCERS 2009, Denver, CO, USA, 2009.

[6] M.S. Kranz, G.K. Fedder, "Micromechanical vibratory rate gyroscopes fabricated in conventional CMOS," in Proc. Symposium Gyro Technology 1997, Stuttgart, Germany, pp. 3.0-3.8., September 16-17, 1997.

[7] A.R. Schofield, A.A. Trusov, A.M. Shkel, "Versatile Sub-mTorr Vacuum Packaging for the Experimental Study of Resonant MEMS," IEEE MEMS 2010 Conference, Hong Kong, January 24-28, 2010.

[8] Y. Xie, S. Li, Y. Lin, Z. Ren, C. Nguyen, "1.52-GHz micromechanical extensional wine-glass mode ring resonators," IEEE Trans. Ultrasonics, Ferroelectrics and Frequency Control, vol.55, no.4, pp. 890-907, April 2008.

[9] S. Lee, J. Cho, S. Lee, M.F. Zaman, F. Ayazi, K. Najafi, “A low power oven-controlled vacuum package technology for high performance MEMS," IEEE MEMS 2009, Sorento, Italy, January 2009, pp. 753756. 